\title{
Features and Model Adaptation Techniques for Robust Speech Recognition: A Review
}

\author{
Kapang Legoh \\ North Eastern Regional \\ Institute of Science \& \\ Technology (NERIST), \\ Department of Computer \\ Science \& Engineering
}

\author{
T. Tuithung \\ North Eastern Regional \\ Institute of Science \& \\ Technology (NERIST), \\ Department of Computer \\ Science \& Engineering
}

\author{
U. Bhattacharjee \\ Rajiv Gandhi University, \\ Department of Computer \\ Science \& Engineering
}

\begin{abstract}
In this paper, major speech features used in state-of-the-art technology in speech recognition research are reviewed. Also a brief review of major technological advancements during last few decades and a trend towards development of robust speech recognition system in terms of feature and model adaptation techniques is given. It has been the dream of researchers to develop a machine that recognizes speech and understands natural language like human but the reality is that the performance of the speech recognition system drastically degrades due to various adverse conditions like noise, variability in speaker, channel, device and mismatches in training and testing. This paper may be useful as a tutorial and review on state-of-the-art techniques for feature selection, feature normalization and model adaptation techniques for development of robust speech recognition system.
\end{abstract}

\section{General Terms}

Automatic Speech Recognition (ASR), Robust ASR, Normalization, Adaptation and Hybrid model.

\section{Keywords}

Spectral, Cepstral Features, Feature Enhancement, Compensations, Model Adaptation and Hidden Markov Model.

\section{INTRODUCTION}

Speech is the primary means of communication. It carries many information apart from the intended meaning for which it is uttered. The information like gender identity, probable age (child or adult), emotions, health conditions of the speaker, direction, contextual meaning, language information etc. are also carried by the speech signal apart from its intended meaning. The desire to develop a machine that understands human speech and interact with human in one's own language has been the driving force for the researches and technological developments in the field of Automatic Speech Recognition (ASR). Although lot of works and progresses have been made by various researchers around the world towards development of robust speech recognition system [1-3], it is still a distant dream to build an Automatic Speech Recognition system (ASR) that not only recognizes speech but also understands the natural language like human being. Various researchers have been working for convergence of technology towards development of universal system that recognizes any speech and robustly perform under different variability and other adverse environments.

There are two major activities in speech recognition research in present day technology: Front end analysis (signal modeling) and statistical modeling. Front end analysis represents the process of converting sequences of speech samples to feature vectors sequence and the statistical modeling is the speech recognition part which can be modeled using Hidden Markov Model (HMM) or hybrid of both of Artificial Neural Network. Different techniques like Gaussian Mixture Model, K-means clustering, Expectation maximization etc. are used for training and testing purposes and Viterbi algorithm is used for decoding of path sequence.

The main purpose of signal modeling is to parameterize the speech signal. It is desired that the parameterizations are robust to noise and variations in channel, device, speaker, session and other adverse environments and capture the spectral dynamics of speech, or changes of the spectrum with time, which is also referred to as the temporal correlation problem. Perceptuallymeaningful parameters along with the delta and double delta features are chosen by various researchers. With the introduction of Hidden Markov modeling techniques that are capable of statistically modeling the time course, of the signal parameters, that incorporate both absolute and differential measurements of the signal spectrum have become increasingly common. The difference in processing time between various signal modeling approaches is now a small percentage of the total processing time. The focus today has shifted towards maintaining high performance and minimizing the number of degrees of freedom. Historically, robustness to background acoustic noise has been a major driving force in the design of signal models [4-10]. As speech recognition technologies have become more and more sophisticated, the recognition system itself now contributes more to the noise robustness problem than the signal model. But, signal models are still the major activities in speech recognition, speaker verification research and speech and language related researches. However, the signal models that are good for one type of application may not necessarily be optimal for another. Hence, it is often difficult to isolate frond end processing of speech signal and system model in the development of robust ASR system. In this paper, different speech features are briefly reviewed and compared for different applications and also feature normalization techniques and model adaptation approaches for obtaining over all robustness in the system are presented.

The paper is organized as follow. Section 2 describes brief comparisons of different speech features for different speech applications, section 3 gives feature normalization techniques, section 4 give a brief overview of model adaptation approaches towards developing robust speech recognition system and finally section 5 gives conclusion of the paper followed by references. 


\section{SPEECH FEATURES AND THEIR COMPARISONS}

Several features can be extracted from speech signal and all may not be important for a particular type of application. For example, an ideal feature for speaker identification would have large speaker variability between speakers but for speech recognition speaker variability must be small or minimum. Apart from this, feature must be robust against various adverse environments, occur frequently and naturally in speech, easy to measure from speech signal, difficult to impersonate/mimic, not affected by the speaker's health or long-term variations in voice etc. For speech recognition, it is required to consider large number of speakers and speech database and apply normalization techniques on the speech features to increase performance accuracy. Moreover, the number of features should also be relatively low to reduce the curse of dimensionality [11] and reduce complexity of computation. There are several categories of features used in speech processing; and different features or combinations of several features are required for different type applications. The main categories of speech features are temporal features (energy, zero crossing rates, power, root mean square of signal, voice onset time (VOT), rise time (RT) etc. ), short-term spectral features (Line Spectral Frequencies (LSF), Linear Predictive Coefficients (LPC Coefficients), Linear Predictive Cepstral Coefficients (LPCC), Mel Frequency Cepstral Coefficients (MFCC), Perceptual Linear Prediction Coefficients (PLPCC) etc.), voiced source features (Fundamental Frequency and related features), spectrotemporal features(first and second order temporal derivatives of features), prosodic features(fundamental frequency (F0), syllable stress, intonation patterns, phone duration, speaking rate, energy distribution and rhythm ) and high-level features [14].

In this paper, the major features like Linear Predictive Coefficients (LPC), Linear Predictive Cepstral Coefficients (LPCC), Mel Frequency Cepstral Coefficients (MFCC), Perceptual Linear Prediction Coefficients (PLPCC) RASTAPLP cepstral coefficients and their temporal derivatives which are most commonly used features in speech recognition research are presented. Since the focus is on feature normalization and model adaptation techniques, the following sections briefly describe these features without giving details of extraction techniques and non-parametric method like vector quantization, VQ. Fig. 1 shows the broad signal processing method and extraction of features.

The cepstral representation of the speech spectrum provides a good representation of the local spectral properties of the signal for a given frame. An improved representation is obtained by extending the analysis to include information about the temporal variation in the speech signal. This is because the temporal information is lost in the frame-by-frame analysis. A common way to incorporate or recover temporal information to features is through time derivatives i.e., first and second-order time derivative estimates, known as delta and double-delta coefficients, respectively $[12,13]$ and append these derivatives in the original cepstral coefficients. However, there are several advantages and disadvantages in using these features for different speech applications. Some major advantages and disadvantages of these features are briefly reviewed and given in Table 1 and Table 2.

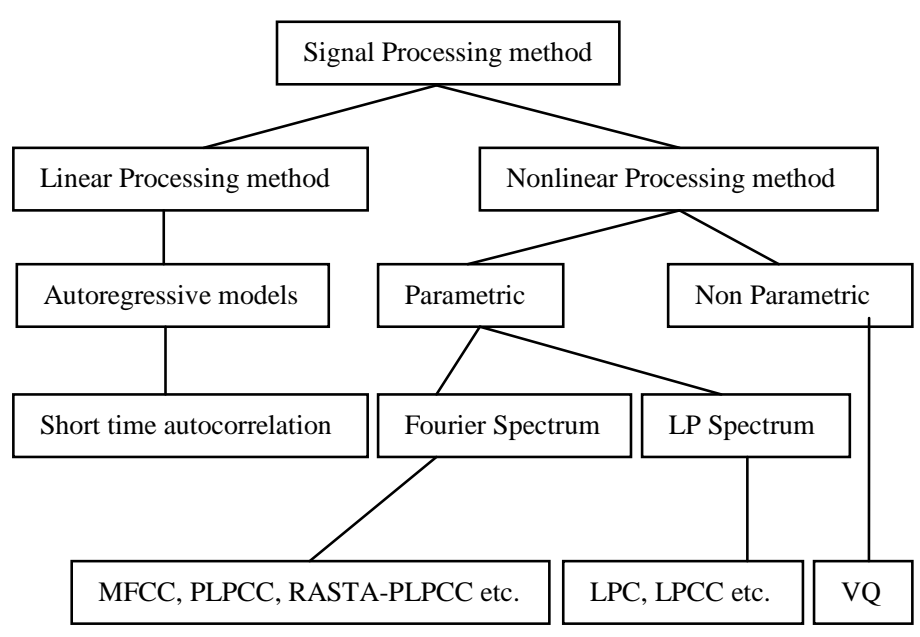

Fig. 1: Signal processing methods for feature extraction.

The goal of speech recognition research is to increase the accuracy and robustness of the ASR systems with respect to changes in the environments. Since variations, adverse environments and mismatches between training and testing conditions lead to a considerable degradation in performance, systems must be retrained for every different environment used. A system is said to be robust if the recognition accuracy for a new environment is the same or higher than that obtained when the system is retrained for that environment. Attaining such performance is a very difficult task for real time application. Different approaches are used by researchers to develop environment-independent ASR system. There are two approaches used, Feature Domain Approach and Model Based Approach, which are presented in the following sections.

\section{FEATURE DOMAIN ADAPTATION APPROACHES}

The aim of feature domain techniques is to make speech features robust and more consistent under different kinds of noise distortions and signal to noise ratios (SNR) by making clean and noisy speech features identical to each other while preserving the discriminative power of the features. The following section briefly describes speech enhancement, feature compensation, feature normalization, and temporal filtering techniques to obtain such robust speech features.

\subsection{Speech Enhancement}

The objective of human listening is quite different from that of automatic speech recognition. For human listening, the objective is to improve the quality and intelligibility of speech signals, while for ASR the objective is to reduce the difference between the clean and noisy speech features. Despite this difference, it is reasonable to assume that the good quality and intelligibility of speech signal usually leads to small mismatch between clean and noisy speech features. The speech enhancement techniques were designed for enhancing noise-corrupted speech signals for human listening and later these techniques have been modified and applied in the feature extraction process of speech recognition systems. Popular speech enhancement techniques are briefly discussed as follows: 
Table 1. Comparisons of feature vectors (LP Coefficients, LPCC, MFFCC, PLP, RASTA-PLP and wavelet)

\begin{tabular}{|c|c|c|}
\hline Features & Advantages & Disadvantages \\
\hline $\begin{array}{c}\text { LPC } \\
\& \\
\text { LPCC }\end{array}$ & $\begin{array}{l}\text { LPC is a production based method and it provides good model of } \\
\text { vocal tract characteristics. It represents the spectral envelope by low } \\
\text { dimension feature vectors and provides linear characteristics. } \\
\text { LPC is analytically tractable model. It is mathematically precise and } \\
\text { straight forward to implement in either software or in hardware. }\end{array}$ & $\begin{array}{l}\text { The LP models the input signal with } \\
\text { constant weighting for the whole frequency } \\
\text { range. However, human perception does } \\
\text { not have constant frequency perception in } \\
\text { the whole frequency range. } \\
\text { Another serious problem with the LPC is } \\
\text { that they are highly correlated but it is } \\
\text { desirable to obtain less correlated features } \\
\text { for acoustic modeling. Other inherent } \\
\text { drawback of conventional } \\
\text { LPC analysis is its inability to include } \\
\text { speech specific a priori information in the } \\
\text { modeling process. }\end{array}$ \\
\hline MFCC & $\begin{array}{l}\text { MFCC is perception based feature. MFCCs are derived from the } \\
\text { power spectrum of the speech signal, while the phase spectrum is } \\
\text { ignored. This is done mainly due to our traditional belief that the } \\
\text { human auditory system is phase deaf, i.e., it ignores phase spectrum } \\
\text { and uses only magnitude spectrum for speech perception. MFCC } \\
\text { features are advantageous as it mimics some of the human } \\
\text { processing of the signal. Characteristics of the slow varying part are } \\
\text { concentrated in the low cepstral coefficients. Individual features of } \\
\text { MFCC are weakly correlated which turns out to be an advantage for } \\
\text { the creation of statistical acoustic model. } \\
\text { MFCC features give good discrimination and lend themselves to a } \\
\text { number of manipulations. It is capable of capturing the phonetically } \\
\text { important characteristics of speech. } \\
\text { Also band-limiting can easily be employed to make it suitable for } \\
\text { telephone applications. It has the basic desirable property that the } \\
\text { coefficients are largely independent, allowing probability densities to } \\
\text { be modeled with the diagonal covariance's metrics. } \\
\text { Mel scaling has been shown to offer better discrimination between } \\
\text { phones, which is an obvious help in recognition. }\end{array}$ & $\begin{array}{l}\text { A small drawback is that MFCCs are more } \\
\text { computationally expensive than LPCC due } \\
\text { to the Fast Fourier Transform (FFT) at the } \\
\text { early stages to convert speech from the } \\
\text { time to the frequency domain. First, they do } \\
\text { not lie in the frequency domain. Secondly, } \\
\text { as most current HMMs use Gaussian } \\
\text { distributions with diagonal covariance } \\
\text { matrices, these HMMs cannot benefit from } \\
\text { cepstral weighting. However, it is well- } \\
\text { known that MFCC is not robust enough in } \\
\text { noisy environments, which suggests that } \\
\text { the MFCC still has insufficient sound } \\
\text { representation capability, especially at low } \\
\text { SNR. } \\
\text { Though MFCCs have been very successful } \\
\text { in speech recognition, they have the } \\
\text { following two problems: (1) They do not } \\
\text { have any physical interpretation, and (2) } \\
\text { Liftering of cepstral coefficients, found to } \\
\text { be highly useful in the earlier dynamic } \\
\text { warping-based speech recognition systems, } \\
\text { has no effect in the recognition process } \\
\text { when used with continuous. The features } \\
\text { derived from either the power spectrum or } \\
\text { the phase spectrums have the limitation in } \\
\text { representation of the signal. }\end{array}$ \\
\hline PLP & $\begin{array}{l}\text { The PLP method takes advantage of three principal characteristics } \\
\text { derived from the psychoacoustic properties of the human hearing } \\
\text { viz., spectral resolution of the critical band, equal loudness curve } \\
\text { adjustment and application of intensity-loudness power law which } \\
\text { make it more effective than LPCC. } \\
\text { It approximates the speaker independent effective second formant. It } \\
\text { emphasizes first two formants F1 and F2 and deemphasizes high } \\
\text { frequencies in contrast with LP which emphasizes high frequencies, } \\
\text { F3. It reduces disparity between voiced and unvoiced speech. PLP } \\
\text { peaks are relatively insensitive to vocal tract length and PLP } \\
\text { estimates are highly correlated with the relatively speaker } \\
\text { independent front of the vocal tract. It reduces the sensitivity of ASR } \\
\text { front ends to changes in high frequencies and increases the } \\
\text { sensitivity to changes in F2 and F1. } \\
\text { It is computationally efficient and it yields a low-dimensional } \\
\text { representation of the speech. It is based on short term spectrum of } \\
\text { the speech. However, PLP technique is vulnerable when the short } \\
\text { time spectral values are modified by the frequency response of the } \\
\text { communication channel. } \\
\text { Computational requirements of PLP are comparable to their } \\
\text { conventional LP analysis. The advantage of the PLP technique over } \\
\text { the conventional LP is that it allows for the effective suppression of } \\
\text { the speaker-dependent information by choosing the particular model }\end{array}$ & $\begin{array}{l}\text { One of weak points of PLP analysis is the } \\
\text { dependency of the result on the overall } \\
\text { spectral balance on formant amplitudes. } \\
\text { The spectral balance is easily affected by } \\
\text { factors such as the recording equipment, } \\
\text { the communication channel or additive } \\
\text { noise. The effect of the overall spectral } \\
\text { balance, to some extent, can be suppressed } \\
\text { a posterior by a proper distortion measure. } \\
\text { Just like most other short-term spectrum } \\
\text { based techniques this method is vulnerable } \\
\text { when the short-term spectral values are } \\
\text { modified by the frequency response of the } \\
\text { communication channel. }\end{array}$ \\
\hline
\end{tabular}




\begin{tabular}{|c|c|c|}
\hline & $\begin{array}{l}\text { order. PLP features are advantageous as it also mimics some of the } \\
\text { human processing of the signal. }\end{array}$ & \\
\hline $\begin{array}{l}\text { RASTA- } \\
\text { PLP }\end{array}$ & $\begin{array}{l}\text { In ASR the task is to decode the linguistic message in speech. This } \\
\text { linguistic message is coded into movements of the vocal tract. The } \\
\text { speech signal reflects these movements. The rate of change of non- } \\
\text { linguistic components in speech often lies outside the typical rate of } \\
\text { change of the vocal tract shape. } \\
\text { The RASTA (relative spectral transform) takes the advantage of this } \\
\text { task. It suppresses the spectral components which change more } \\
\text { slowly or quickly than the typical range of change of speech. It } \\
\text { improves the performance of a recognizer in presence of convolution } \\
\text { and additive noise. } \\
\text { It is also used for enhancement of noisy speech. It makes the } \\
\text { recognizers much more robust to factors like choice of the } \\
\text { microphone or even to the microphone position relative to the mouth } \\
\text { that compare to short term spectrum based methods. } \\
\text { RASTA was developed to make PLP more robust to linear spectral } \\
\text { distortions. It takes advantage of the fact that the temporal properties } \\
\text { of environmental effects such as noise, distortions and convolution } \\
\text { are quite different from the temporal properties of speech. } \\
\text { The RASTA filter can be used either in the log spectral or cepstral } \\
\text { domains. In fact the RASTA filter band passes each feature } \\
\text { coefficient. } \\
\text { Linear channel distortions appear as an additive constant in both the } \\
\text { log spectral and the cepstral domains. The high-pass portion of the } \\
\text { equivalent band pass filter alleviates the effect of convolutional noise } \\
\text { introduced in the channel. RASTA-PLP is quiet efficient in dealing } \\
\text { with convolution noise. It is advantageous where the channel } \\
\text { conditions are not known a priori or where the channel conditions } \\
\text { may change unpredictably during the use of the recognizer. }\end{array}$ & $\begin{array}{l}\text { A possible shortcoming of the non } \\
\text { inclusion of Linear Discriminant Analysis } \\
\text { (LDA) in RASTA-PLP is that the } \\
\text { quantization effect took effect before the } \\
\text { computation of LDA matrices. } \\
\text { LDA attempts to maximize the linear } \\
\text { separability between data points belonging } \\
\text { to different classes in the low-dimensional } \\
\text { representation of the data. }\end{array}$ \\
\hline Wavelet & $\begin{array}{l}\text { Due to the efficient time frequency localization and the multi- } \\
\text { resolution characteristics of the wavelet representations, the wavelet } \\
\text { transforms are quite suitable for processing non stationary signals } \\
\text { such as speech. } \\
\text { In wavelet analysis one can look signals at different scales or } \\
\text { resolutions: a rough approximation of the signal might look } \\
\text { stationary, while at detailed level discontinuities become apparent. } \\
\text { One major advantage afforded by wavelets is the ability to perform } \\
\text { efficient localization in both time and frequency. } \\
\text { The multi-resolution property of wavelets that can decompose the } \\
\text { signal in terms of the resolution of detail makes analysis capable of } \\
\text { revealing aspects of data that other signal analysis techniques miss } \\
\text { aspects like transients, breakdown points, discontinuities in higher } \\
\text { derivatives and self similarity. } \\
\text { Wavelet analysis can often compress or de-noise a signal without } \\
\text { appreciable degradation. Wavelets can zoom in to time } \\
\text { discontinuities and those orthogonal bases, localized in time and } \\
\text { frequency can be constructed. } \\
\text { Wavelet transforms have advantages over traditional Fourier } \\
\text { transforms for representing functions that have discontinuities and } \\
\text { sharp peaks, and for accurately deconstructing and reconstructing } \\
\text { finite, non-periodic and/or non-stationary signals. } \\
\text { Hence, wavelet transform is well suited to transient signals whose } \\
\text { frequency characteristics are time varying, especially like speech. }\end{array}$ & $\begin{array}{l}\text { Wavelet method is non-adaptive because } \\
\text { the same basic wavelets are used for all } \\
\text { data. }\end{array}$ \\
\hline
\end{tabular}

Table 2. Different features, their parameters for different speech applications.

\begin{tabular}{|l|l|}
\hline \multicolumn{1}{|c|}{ Types of Recognition } & \multicolumn{1}{c|}{ Features } \\
\hline Isolated digit recognition & MFCC + Energy + Derivatives \\
\hline CSS & MFCC + Energy + Derivatives \\
\hline IWCSR & LP Coefficients, LPCC + Derivatives \\
\hline RCSR & LPC + Derivatives \\
\hline CWR & MFCC + Energy + Derivatives \\
\hline CSR & LPCC + Derivatives \\
\hline IWR & MFCC + Derivatives \\
\hline
\end{tabular}




\begin{tabular}{|l|l|}
\hline RSR isolated and connected & MFCC + Energy + Derivatives \\
\hline LVCSR & MFCC + Energy + Derivatives \\
\hline Phoneme Recognition & MFCC + Energy + Derivatives \\
\hline Isolated noisy Digit recognition & MFCC + Energy + Derivatives or RASTA-PLP \\
\hline NSR & MFCC + Energy + Derivatives + RASTA-PLP \\
\hline RSSR in noisy (IWR/CS) & MFCC + LPC + Zero Crossing with peak amplitudes or RASTA-PLP \\
\hline SSR & MFCC + Derivatives \\
\hline
\end{tabular}

Abbreviations used: CSS: Continuous Spontaneous Recognition, IWCSR: Isolated Word continuous Speech Recognition, RCSR: Robust Continuous Speech Recognition, CWR: Continuous word Recognition, CSR: Continuous Speech Recognition, IWR: Isolated Word Recognition, RSR: Robust Speech Recognition, LVCSR: Large Vocabulary Continuous Speech Recognition, NSR: Noisy Speech Recognition, RSSR: Robust Spontaneous Speech Recognition, SSR: Spontaneous Speech Recognition [102].

\subsubsection{Spectral Subtraction}

Spectral subtraction is a simple but an effective way to reduce additive noise's effects in speech signal. Spectral subtraction estimates the clean speech spectrum by subtracting the estimated additive noise spectrum from the noisy speech spectrum $[20$, 22]:

$$
|\hat{X}(k)|^{2}=|Y(k)|^{2}-|N(k)|^{2}
$$

where $|\hat{X}(k)|^{2}$ is the estimated clean speech spectrum, $\mid\left(\left.Y(k)\right|^{2}\right.$ is the observed noisy speech spectrum, $|N(k)|^{2}$ is the estimated noise spectrum, and $k$ is the frequency bin index. Spectral subtraction is motivated by the fact that the noise corruption is additive in the power spectrum domain, in expected sense if the noise has zero mean in time domain, and is assumed to be independent from the speech, i.e.

\section{$E\left[|Y(k)|^{2}\right]=E\left[|X(k)+N(k)|^{2}\right]=E\left[|X(k)|^{2}\right]+E\left[|N(k)|^{2}\right]$}

where $E[]$ denotes expected value. The performance of spectral subtraction is directly affected by the accuracy of noise estimation, which is a very difficult task by itself. In some cases, the noise estimate $|N(k)|^{2}$ is even larger than the noisy speech $|Y(k)|^{2}$, hence the estimated clean spectrum will be negative. When this happens, the value of clean spectrum is usually set to zero. This simple solution results in non natural spectral vectors and causes "musical noise" phenomenon, which is annoying for human listening and degrades speech recognition performance. There are several kinds of spectral subtraction, and they mainly differ in the way to handle the "musical noise". In [23], over subtraction and spectral floor are used to provide a tradeoff between the "musical noise" and residual noise level. In [24], it is proposed to apply the masking properties of ear to determine the amount of subtraction. The basic idea is that for those noise components that cannot be heard by a human ear, it is not subtracted, so there is a smaller amount of subtraction and therefore smaller degree of distortion. Despite its application in enhancing speech for hearing, spectral subtraction has also been used to preprocess noisy speech in the feature extraction process of speech recognition systems [25]. The limitation of spectral subtraction is that it aims to reduce noise distortion in the signal domain and has no direct relationship with the final speech recognition task, i.e. to achieve high recognition accuracy. In addition, spectral subtraction's performance depends heavily on the accuracy of noise estimation which is difficult especially when the noise is non-stationary and has similar characteristics as speech signal, such as babble noise.

\subsubsection{MMSE Spectral Magnitude Estimator.}

A more advanced speech enhancement technique than spectral subtraction is the optimal estimator of speech's short-time spectral amplitude (STSA) in the minimum mean square error (MMSE) sense [27, 28]. In the MMSE STSA estimator, the phase and amplitude of spectral components of clean speech signal and noise are assumed to be independent Gaussian variables. With this assumption, the distribution of the spectral component of noisy speech signal follows the Rayleigh's distribution. The MMSE estimate of the clean spectral amplitude is then derived based on these models, and the resulting solution of the estimator is a function of the a priori SNR and the a posteriori SNR of the speech signal. The a priori SNR is the expected SNR before the current frame is observed. It is critical for the performance of MMSE STSA estimator and can be estimated using either Maximum Likelihood estimation or a "decision-directed" method. The a posteriori SNR is the instantaneous SNR of the current frame. Several methods have been proposed to improve the accuracy of the estimate of the a priori SNR. Some researchers focused on improving the average weighting parameter $\alpha$ of the decision directed method [27, 28], which controls the speed of adaptation. Soon and Koh [29] proposed to estimate $\alpha$ from the changing speed of frame energy. This idea is further extended in [30] by using a frequency-dependent MMSE estimator of $\alpha$. Besides the estimation of $\alpha$, to incorporate more information, Israel [31] proposed a non causal a priori SNR estimator that employs both past and future frames for better estimation. Another approach by $\mathrm{Hu}$ and Loizou [32] reduces the variance of the a priori SNR estimate indirectly by reducing the variance of noise estimate. Another important characteristic of the MMSE STSA estimator that incorporates a signal absence probability (SAP) was introduced by McAulay and Malpass [21]. With SAP, the estimate of a clean speech becomes:

$$
\hat{X}(k)=P(\text { Speech present } \mid Y(k)) \hat{X}_{\text {MMSE }}(k)
$$

where $P($ speech present $\mid Y(k))$ is the posterior probability of speech present at the $k^{\text {th }}$ frequency bin when noisy spectral coefficient $Y(k)$ is observed, and $\hat{X}_{M M S E}(k)$ is the MMSE estimation of clean spectral coefficients. Later, the MMSE estimator of STSA was extended to log spectral domain [28] to simulate the nonlinear compression of the human auditory system. The MMSE STSA have been successfully applied to noisy speech recognition tasks, e.g. in [33, 34].

\subsubsection{Subspace-based Techniques.}

Another popular speech enhancement technique is the signal subspace method, which is motivated by the fact that noisy speech signal can usually be decomposed into two subspaces: the signal plus noise subspace and the noise only subspace. During the enhancement process, the noise only subspace can be removed completely and the clean speech signal can be estimated from the signal plus noise subspace. There are two methods to decompose the noisy signal into the two subspaces, namely the singular value decomposition (SVD) method and the Karhunen-Loeve transform (KLT). In the SVD-based method [35], the clean signal is reconstructed from the singular vectors corresponding to the largest singular values. It is believed that the singular vectors corresponding to the largest singular values 
contain speech information, while the singular vectors corresponding to the smallest singular values contains noise information. This approach provides large SNR gains for speech corrupted by white noise. In the Quotient SVD-based approach proposed by Jensen et al [36], the previous approach is extended to suppress colored noise. However, QSVD is computationally expensive.

Many approaches also use KLT to decompose noisy signal. In Ephraim and Van Trees' method [37], the estimator minimizes speech distortion subject to a given residual noise level constraint. In this way, a mechanism is provided to adjust the tradeoff between the signal distortion and the residual noise level. Huang and Zhao [38] extended the method of Ephraim and Van Trees by proposing an energy-constrained signal subspace method (ECSS). The idea was to match the short-time energy of enhanced speech signal to the unbiased estimated of the clean speech. They declared that this method recovered the low-energy segments in continuous speech effectively. Rezayee and Gazor [39] proposed an algorithm to reduce colored noise by diagonalizing the noise correlation matrix using the estimated Eigen values of the clean speech and nulling any off-diagonal elements. Mittal and Phamdo [41] also extended method of Ephraim and Van Trees to colored noise by providing proper noise shaping for colored noise without pre-whitening. One important assumption of signal subspace approach is that the largest singular values or Eigen values are from speech and the smallest values are from noise. In [40] several subspace-based methods have been evaluated on noisy speech recognition task.

\subsection{Feature Compensation}

Speech enhancement techniques try to recover the time domain speech signal for human hearing but in feature compensation methods the aim is to recover clean speech coefficients from noisy speech coefficients during the feature extraction process of speech recognition without generating corrected speech signal in time domain. There are another two major differences between these two groups of methods. One difference is that speech enhancement techniques usually operate in time domain, frequency domain or $\log$ frequency domain, while feature compensation methods usually work in the log filter bank domain or cepstral domain. Another difference is that feature compensation methods are solely designed for noisy speech recognition tasks, while speech enhancement methods are originally proposed to improve speech signal for human listening. Feature compensation methods can be classified into two groups based on whether they use the environment model described in the following sections: i.e. model-based approach and data-driven approach.

\subsubsection{Model-based Feature Compensation}

An early model-based feature compensation technique is the code-dependent cepstral normalization (CDCN) [42]. The clean cepstral vector $x$ are modeled by a Gaussian mixture model (GMM). The CDCN estimates the clean cepstral vectors from the noisy observations in the MMSE sense. The closed-form solution of the MMSE estimator of $\mathrm{x}$ is:

$$
\hat{x}=y-\hat{h}-\sum_{i=1}^{M} p(i \mid y) \hat{r}(i)
$$

where $\hat{x}$ and $\hat{h}$ are the estimate of the clean cepstral vector and channel distortion, respectively. $y$ is the noisy cepstral vector, $p(i \mid y)$ is the posterior probability of the $i^{\text {th }}$ mixture in the GMM after $y$ is observed, $M$ is the number of mixtures, and $\hat{r}(i)$ is the codeword-dependent correction vectors that need to be estimated. In CDCN phase insensitive environment model is used. It is to be observed that the GMM of the clean cepstral vector $x$ are first adapted to noisy GMM using estimated noise and channel distortions and environment model, then $p(i \mid y)$ for all mixtures are calculated.

The noise and channel distortions are estimated using the ML criterion as there is usually no prior information about them available. The distortions are assumed to be constant during the analysis duration, e.g. an utterance. By assuming speech frames to be independent from each other, the log likelihood of the training data is:

$\log p(Y \mid n, h)=\sum_{t=1}^{T} \log p\left(y_{t} \mid n, h\right)$

where $Y=\mathrm{y}_{1}, \ldots \mathrm{y}_{\mathrm{T}}$ is the feature vector sequence of an utterance, $n$ is the noise distortion and $T$ is the number of frames in the utterance to be processed. In [42], the distribution $p(y \mid n, h)$ is obtained by using the environment model and the distribution of $x$ with some assumptions. The optimization is implemented using the expectation maximization (EM) algorithm [43].

Another model-based feature compensation method is proposed by Deng et. al. in [44 - 46]. A major difference between Deng's estimator and $\mathrm{CDCN}$ is that the phase sensitive environment model is used in Deng's estimator for more accurate modeling of speech-noise relationship. Besides, Deng's estimator operates in the $\log$ Mel filterbank domain, while $\mathrm{CDCN}$ is a cepstral coefficients estimator. Similar to CDCN, there are two major parts in Deng's estimator, i.e. the MMSE estimation of the clean speech feature vector based on the adopted environment model and the prior probability distribution of clean speech, and the estimation of noise distortion. The GMM is also used for clean feature vector modeling in Deng's estimator. The phase factor is also modeled as zero-mean Gaussian distributed. With this prior distribution of clean speech, phase, and the phase-sensitive environment model, the MMSE estimator of clean feature vector can be obtained. However, the estimator is too complex and needs to be simplified by using the second-order Taylor series expansion. Channel distortion is ignored and sequential noise estimation [20] is used to track additive noise. In addition, the assumption of stationary noise in CDCN is removed in Deng's estimator. It was found by Deng et. al. that the recognition performance of the phase-sensitive MMSE estimator [44] was better than that of the phase-insensitive MMSE estimator in [48] with $54 \%$ error rate reduction. This shows that the incorporation of the phase information benefits the feature compensation process by including relevant information. If the phase factor is set to zero, the phase-sensitive MMSE estimator degenerates to spectral subtraction. Later, the phase-sensitive MMSE estimator is expanded to include the first order derivatives of the speech features in the log Mel filterbank domain [45], due to the assumption that the strong dynamic property of speech features are important for the enhancement of the features. The static and dynamic features are assumed to follow a GMM distribution and be independent from each other. Then the noisy speech feature distribution function is derived and the clean speech features are estimated using the MMSE criterion. The work of Deng et. al. was further expanded by incorporating a feature compensation uncertainty [46] in the decoding process. The feature compensation uncertainty accounts for the deviation of the enhancement feature from the clean feature, i.e. the variance of the feature estimator. To better decode the noisy speech, this uncertainty should be taken into account in the decoding process. One way to do this is to integrate the acoustic score 
over this uncertainty space, i.e. over all possible clean feature values. One issue for incorporation of the uncertainty is how to efficiently calculate the integration. The integration is effectively the same as adding the variance of the feature estimator (the uncertainty) to the Gaussian's of the HMM states if the feature estimation error is assumed to be zero-mean Gaussian distribution [46]. Another issue is how to effectively estimate the feature estimator's variance. In [19], analytical solutions are derived by making use of the phase-sensitive environment model.

\subsubsection{Data-driven Feature Compensation}

The simplest data-driven feature compensation is the cepstral mean normalization (CMN) [12]. In CMN, the features are compensated as:

$\hat{x_{t}}=y_{t}-r$

where $\hat{x}_{t}$ and $y_{t}$ are the estimated clean feature vector and noisy feature vector for the $t^{\text {th }}$ frame respectively, and $r$ is the correction term that is the mean of the features, usually obtained by averaging the feature vectors over an utterance. The mean of features is in fact the optimal estimate of the correction term $r$ in the MMSE sense if only a single correction vector is allowed [49]. The operation of CMN to compensate all feature vectors by a single fixed correction vector is too limiting. The use of a single vector $r$ can only compensate for convolutional noise in the feature domain. In [50], a method called multivaRiate gAussian-based cepsTral normaliZation (RATZ) is proposed to use multiple correction vectors. In RATZ, the clean feature space is modeled by a GMM. The distribution of the noisy speech is also assumed to be GMM. It is observed that in the log Mel filterbank and cepstral domain, the effect of noise on the distribution of speech signal is that the mean is shifted and the variance is either decreased or increased depending on the SNR. Therefore, the noisy GMM can be approximated by adding a correcting term to the mean and variances of the clean GMM. Let the distribution of the cepstral vectors of the clean and noisy speech be GMM with the same number of mixtures

$$
\begin{aligned}
& p(x)=\sum_{i=1}^{M} p(i) N_{x}\left(\mu_{x}^{i}, \sum_{x}^{i}\right) \\
& p(y)=\sum_{i=1}^{M} p(i) N_{y}\left(\mu_{y}^{i}, \sum_{y}^{i}\right)
\end{aligned}
$$

where $\mu_{y}^{i}, \sum_{y}^{i}$ represent mean and variance of vectors. The noisy distribution function can be approximated by adding correction terms to the clean mean and covariance parameters

$$
\begin{aligned}
& \mu_{y}^{i}=\mu_{x}^{i}+r^{i}, \quad i=1, \ldots ., M \\
& \sum_{y}^{i}=\sum_{x}^{i}+R^{i}, \quad i=1, \ldots ., M
\end{aligned}
$$

The correction terms are estimated based on the maximization of the likelihood for the noisy observation. As there is no closedform solution for the correction terms, the EM algorithm is applied again. After the $r^{i}$ and $R^{i}$ are obtained, the RATZ estimates the clean cepstral vector using the MMSE criterion as follows:

$$
\hat{x}=y-\sum_{i=1}^{M} p\left(i \mid y, \mu_{y}^{i}, \sum_{y}^{i}\right) r^{i}
$$

which is a weighted sum of mean correction vectors $r^{i}$. The correction vectors $r^{i}$ and $R^{i}$ can also be used to adjust the parameters of acoustic models for better match between the model and noisy data. The estimated noisy mean and variance are used to evaluate the posterior of mixtures. For more detail, interested reader may refer [51-54].

\subsection{Feature Normalization}

Unlike speech enhancement and feature compensation methods that aim to recover the clean speech coefficients, the feature normalization methods normalize the speech coefficients, usually cepstral coefficients, to a new space where the noise distortion is reduced. It should be mentioned that both the compensation and normalization methods modify feature vectors and thus the difference between them is not very clear, however, feature normalization methods usually modify certain statistics of features, e.g. global means and variances, to some reference values which are usually obtained from clean speech or simply pre-defined values. A rationale of doing so is that the statistics of speech features are changed when speech signal is distorted by noise. By normalizing the statistics of the speech features, it is expected that some systematic distortion caused by noise wil be reduced. In this section, major feature normalization methods are reviewed.

\subsubsection{Cepstral Mean and Variance Normalization}

A simple and effective feature normalization method is the cepstral mean normalization (CMN), also called cepstral mean subtraction (CMS) $[12,55]$. The CMN is already introduced as a data-driven feature compensation method in above section. However, it can also be treated as a feature normalization method. CMN subtracts the features' mean values from the features. After subtraction, all the feature dimensions will have a zero mean. It is known to be able to reduce the convolutional noises, such as microphone mismatch and linear transmission channels distortion. This is because convolutional noises becomes multiplicative in the frequency domain and additive in the log filterbank and cepstral domain. If the convolutional noise is fixed, it causes a constant shift in the log filterbank and cepstral domain. Therefore, by subtracting the mean from the feature for both clean and noisy speech, the convolutional noise can be removed in theory. The basic $\mathrm{CMN}[12,55]$ estimates the sample mean vector of the cepstral vectors of a sentence and then subtract this mean vector from every cepstral vector of the sentence. An augmented cepstral normalization method [42] estimates the mean vectors for the silence and speech segments of the sentence separately and achieved better results. Instead of using a hard decision on whether a frame is silence or speech, one improvement suggests the use of the a posteriori probability of the frame of being silence $p(n)$, which is similar to the speech absence probability used in the MMSE STSA. The final mean vector is the weighted sum of the silence mean and speech mean, with the weights be $p(n)$ and $1-p(n)$ respectively. In another study [56], CMN is also used together with microphone array and is called position-dependent CMN. The speaker's position is first estimated by the microphone array, and then a pre-trained feature mean for the location is used to perform $\mathrm{CMN}$. In general, the advantage of CMN is its simplicity, low computational cost and easy to be implemented. However, its performance is limited as it uses very few items of prior information about speech and noise, and the use of a single compensation vector provides very little flexibility. Besides mean normalization, the cepstral variance normalization $(\mathrm{CVN})$ [57] normalizes the variances of features to unity. It is well known that noise distortion can change the variance of speech features. At different SNR levels, the variance of features may 
be very different. The CVN is similar to a dynamic gain control. It normalizes the total power of feature trajectories to reduce the difference among features of different environmental conditions. In practice, CMN and CVN is normally used in cascade and called the mean and variance normalization (MVN).

\subsubsection{Histogram Normalization}

Histogram Equalization (HEQ) is also used for normalization. While CMN and CVN normalize the first and second moments of features respectively, histogram equalization [58, 59] normalizes the histogram of the features, i.e. the probability density function (p.d.f.) of the features. Originally used in image processing to automatically balance the contrast of images, HEQ is a technique that can change the histogram of any random variable to match any other desired histogram. For a random variable $x$ with known cumulative distribution function $(c d f) C_{x}$, we can change its $c d f$ to $C_{y}$ by performing

$$
y=C_{y}^{-1}\left(C_{x}(x)\right)
$$

where $y$ is the transformed version of $x$. In speech recognition systems, HEQ can be applied to normalize the distribution of speech features. A reference histogram is first learnt from the training data of acoustic model. Then the histogram of the test features is normalized towards this reference histogram. The process is performed on a dimension-dependent and utterance wise basis. Besides histogram of training data, common probability distributions can be used, such as Gaussian distribution, as the reference. Usually, both the training and testing features are processed by HEQ. HEQ can be seen as a generalization of $\mathrm{CMN}$ and $\mathrm{CVN}$, since when the histogram (p.d.f.) of features are normalized, all moments should be normalized. From another viewpoint, $\mathrm{CMN}$ and CVN provide a linear transformation of the features, while HEQ is able to transform the features nonlinearly.

The CMN, CVN/MVN, and HEQ all have two assumptions, i.e. the assumption that the noise distortion does not change the order statistics of feature trajectories within an utterances or segment; and global statistics of an utterance match that of the whole training set. The order statistics of a feature trajectory refers to which element of the trajectory has the highest value, the second highest value, and so on. In fact, noise distortion usually breaks this order. Hence, even if we can normalize the histogram of the noisy trajectory to the histogram of corresponding true clean trajectory, the normalized trajectory won't be the same as the clean trajectory. Besides this assumption, the assumption about matched statistics is also violated in real situations. In training data, we have a balanced proportion of all the phonemes. However, during testing, as there is very limited number of phonemes in an utterance, the phoneme composition of an utterance may be quite different from that of the training set. Hence, it is coarse to normalize the histogram of just one utterance to the global histogram of the entire training set which usually consists of thousands of utterances. There is another simple example to demonstrate the drawbacks of CMN, CVN, and HEQ [61]. Suppose there is an utterance with several words. If several silence frames are appended in both the front and end of the utterance, another utterance is obtained. Although the acoustic content of these two utterances are exactly the same, their normalized versions by CMN, CVN/MVN, and HEQ will be different due to the different proportions of silence frames in the two utterances. The violated assumptions of CMN, CVN, and HEQ are alleviated by the use of cluster based normalization techniques. In [60], a more general solution is proposed, i.e. the class-based HEQ. In this method, the clean training feature vectors are first clustered, and then a reference histogram is estimated for each cluster. During recognition, the noisy feature vectors are first classified into clusters, and then the conventional HEQ is performed for each cluster independently. With class-based HEQ, it is possible that the order statistics of the normalized feature vectors will be different from that of the original vectors. Although this does not guarantee that the order statistics of the normalized features will be more like the corresponding clean features.

\subsection{Temporal Filtering}

Filtering of feature trajectories is also a popular approach to improve the robustness of speech recognition against noise corruption. Typically, the filtering is applied to the trajectories of log filterbank coefficients or cepstral coefficients, which are treated as time domain signals. The filters are usually called temporal filters. The most significant difference between temporal filters and previous feature domain methods are that the temporal filters modify the correlation of features, i.e. second order statistics of features or modulation spectrum, while previous methods modify the probability distribution of features, i.e. first order statistics of features.

A common temporal filtering technique is the extraction of delta and acceleration features [95]. The delta and acceleration (delta-delta) features are generated using the expression

$$
\dot{x}(t) \equiv \frac{d}{d t} x(t) \approx \frac{\sum_{m=-M}^{M} m x(t+m)}{\sum_{m=-M}^{M} m^{2}}
$$

where $2 M+1$ is the number of frames considered in the evaluation. The same formula can be applied to the first order derivative to produce the second order derivative. The delta features are seen as band-pass filtered versions of the static features. This can be seen as a finite impulse response (FIR) filter. The delta filter is a band-pass filter with the center of passband near $15 \mathrm{~Hz}$ modulation frequency. Delta and acceleration features are usually appended to the static features and they are shown to improve the performance of speech recognition significantly. The CMN discussed above, can also be treated as a temporal filter. Strictly speaking, the magnitude response of CMN is time-varying and can only be roughly seen as a highpass fillter. CMN eliminates the very low frequency components of feature trajectories that could be caused by but not limited to channel distortions.

The first commonly used temporal filter specifically designed to reduce the effect of channel distortion and additive noise is the RASTA filter (relative spectra) [96]. The RASTA filter is an IIR filter whose transfer function is defined as:

$$
H_{R A S T A}(z)=0.1 z^{4} \frac{2+z^{-1}-z^{-3}-2 z^{-4}}{1-p z^{-1}}
$$

where $p$ is a parameter controlling the cut-off frequency of the high-pass portion of the filter. Typically, $p$ is set to either 0.98 or 0.94 [96].. It is a bandpass filter that removes the very low frequency and high frequency components of feature trajectories. The design agrees with research findings that speech modulation frequency of $1-16 \mathrm{~Hz}$ is most important for both human and automatic speech recognition [62-68]. RASTA and CMN are both able to reduce channel distortions, and they can be used in concatenation to produce better results.

Another well-known temporal filter designed to reduce feature variation is the autoregressive moving average (ARMA) filter used in the MVA processing [97]. The ARMA filter is defined as:

$y(t)=\frac{y(t-M)+y(t-M+1)+\ldots+y(t-1)+x(t)+\ldots+x(t+M)}{2 M+1}$ 
where $x(t)$ and $y(t)$ are the input and output of the filter, $M$ is the order of the filter. The transfer function is:

$$
H_{A R M A}(z)=\frac{z^{M}}{2 M+1}=\frac{1+z^{-1}+\ldots+z^{-M}}{1-\left(z^{-1}+z^{-2}+\ldots+z^{-M}\right) /(2 M+1)}
$$

The larger the filter orders $M$, the lower the cut-off frequency of the ARMA filter. The optimal value of $M$ is usually dependent on the task. Besides the empirically designed temporal filters such as RASTA and ARMA, some researchers also propose to use data-driven methods for filter design [69-73]. The filters are usually designed from some training data which can be both clean and noisy. Typically, discriminative criteria are used to guide the filter design, e.g. linear discriminative analysis (LDA) and minimum classification error (MCE). Besides, principle component analysis has also been used. The filter parameters are estimated by optimizing the objective function of these criteria. The resulting filters are mostly low-pass or band-pass, similar to RASTA and ARMA filters.

Most of current temporal filters are fixed after being designed. They are not able to track the changes of signal condition during speech recognition.

\section{MODEL ADAPTATION TECHNIQUES}

In contrast to feature domain methods that aim at making features more consistent in various environmental conditions, the model adaptation methods adapt acoustic model to make it better fit to the noisy acoustic environment. Several model adaptation techniques are briefly reviewed and compared in the following sub sections. These methods are grouped into datadriven-based adaptation and environment-model-based adaptation based on whether environment model is used or not.

\subsection{Data-driven-based Adaptation}

There are several data-driven based adaptations like STAR, Stochastic Mapping, MAP and MLLR, and Ensemble modeling. They are briefly reviewed in the following sections.

\subsubsection{STAR.}

The STAR algorithm of Moreno [50] is closely related to the RATZ feature compensation algorithm described in section 3.2. Feature compensation methods usually have a model adaptation counterpart. The basic concept of STAR is similar to that of RATZ. However, unlike RATZ which uses a separate GMM for the prior distribution of clean speech, STAR utilizes the HMM. STAR estimates the correcting terms, $\mu_{\mathrm{k}}$ and $\sum_{\mathrm{k}}$, for the 256 Gaussians using the same way as RATZ, and then compensates the clean mean and variance vectors to approximate the noisy speech distribution [50]. As these Gaussians are shared by all HMM models, once they are compensated, all the HMM states are adapted.

\subsubsection{Stochastic Mapping}

In stochastic matching, it is assumed that the matched acoustic model can be adapted from the clean-trained acoustic model by

$\wedge_{y}=G_{\eta}\left(\wedge_{x}\right)$

where $G_{\eta}()$ is the transformation function and $\eta$ is its parameters. In [74], the transformation function is assumed to be:

$$
\mu_{y}=\mu_{x}+\mu_{b}
$$

$$
\sum_{y}=\sum_{x}+\sum_{b}
$$

where $\mu_{\mathrm{b}}$ and $\sum_{\mathrm{b}}$ are the correction mean and variance and they are estimated for every Gaussian in the acoustic model. In RATZ and STAR, the same type of correction vectors is assumed to estimate noisy speech distribution from clean distribution. The difference is that in stochastic matching, the model is HMM rather than GMM. Similar to feature space stochastic matching, the correction vectors are estimated by maximizing the likelihood of noisy utterance

$$
\hat{\eta}=\arg \max _{\eta} p\left(Y \mid \eta, \wedge_{x}\right)=\arg \max _{\eta} \sum_{S} \sum_{C} p\left(Y, S, C \mid \eta, \wedge_{x}\right)
$$

where $\wedge_{\mathrm{x}}$ is the HMM-based acoustic model trained from clean features, $Y$ is the collection of feature vector for an utterance, $S$ and $C$ are all possible state sequences and mixture sequences of $Y$. The optimization can be solved by EM algorithm. The stochastic matching was improved later by using nonlinear mapping function in [75] and by an SNR-incremental stochastic matching in [76].

\subsubsection{MAP and MLLR}

Another two model adaptation methods, the maximum likelihood linear regression (MLLR) [98] and maximum a posteriori (MAP) [77, 78], have been originally designed for adapting speaker independent acoustic models to a specific test speaker. Due to the similarity between the speaker adaptation and environment adaptation, they are also used for noise robust speech recognition. The MAP approach adapts the acoustic model by optimally using the prior information in the clean trained acoustical model and the posterior information in the noisy observations. The observations are recognized by speech recognition, and only those with high acoustic likelihood score are used for adaptation. The Bayesian adaptation framework used in the MAP approach enables the optimal use of the noisy observations in model adaptation. When the adaptation data are few and the posterior information is weak compared to the prior HMM acoustical models, the models are not adapted much. As there are more and more adaptation data, the models becomes asymptotically equivalent to the ML estimate from noisy observations, which provides optimal decision rule on the test data. However, this adaptation process is quite slow, as only the model parameters directly related to the adaptation data are adapted. In real applications, the adaptation data are few and hence it is necessary to reduce the number of model parameters needed to be adapted. To achieve good adaptation performance, MLLR [98] uses the parameter sharing strategy, i.e., the similar models are tied together and their parameters are adapted together. The degree of model tying is high if the available amount of adaptation data is low and vice versa. For very few data, a global transforms strategy may be used. The basic MLLR adapts the mean vectors of the Gaussian by multiplying it with a transform matrix, which is obtained using maximum likelihood criterion and EM algorithm. The models tied together share the same transformation matrix. The advantage of the MLLR is its ability to provide good adaptation even if data are few. However, MLLR has poor asymptotic properties, which leads to the fast saturation of performance gain with increased data. Researchers found that the MLLR usually outperforms the MAP if the adaptation data are few, but MAP adapts the models better when there are a lot of data and the combination of the two methods yields better performance.

\subsubsection{Ensemble Modeling}

In practice, there is often very little or no data for supervised adaptation. Hence, it is important to reduce the number of free parameters that need to be estimated during adaptation. In the eigenvoice-based speaker adaptation method [81], the number of free parameters is reduced to about 10 such that these parameters can be estimated from limited data robustly. The ensemble modeling can be seen as a generalization of the 
eigenvoice approach for the environment-adaptation problem $[79,80]$. In the ensemble modeling approach, an ensemble of acoustic models are trained using speech data of various environment conditions, e.g. different noise and SNR combinations. After obtaining $P$ acoustic models, the mean vectors of Gaussians in each model are concatenated to form a supervector and there are totally $P$ supervectors, one supervector for one acoustic model. Each supervector has $M \quad x \quad D$ dimensions, where $M$ is the number of Gaussian mixtures in an acoustic model and $D$ is the feature dimension. The idea is to estimate a supervector from these $P$ supervectors based on the noisy observations. The estimated supervector can then be used to construct an acoustic model for speech recognition. In its most general form, the test supervector can be estimated as follows:

$$
\hat{\boldsymbol{S}}_{\text {test }}=\sum_{p=1}^{P} A_{p} \boldsymbol{s}_{p}+b
$$

where $\hat{\boldsymbol{s}}_{\text {test }}$ is the estimated supervector that is supposed to be matched with the noisy test data, $s_{p}$ and $A_{p}$ is the $p$ th supervector and transformation matrix, and $b$ is a correction vector. The transformation matrices $A_{p}, p=1, \ldots, P$ and the correction vector $\mathrm{b}$ can be estimated using the maximum likelihood criterion and the noisy test data. If there is only one model in the ensemble, i.e. $P=1$, the approach degenerates to MLLR. A problem with this general form is that there are too many parameters and it is very difficult if not impossible to estimate these parameters robustly. A practical form of ensemble modeling approach [79, 80] is as follows:

$$
\hat{\boldsymbol{s}}_{\text {test }}=\sum_{p=1}^{P} w_{p} s_{p}
$$

where $w_{p}$ is a scalar weight rather than a transformation matrix. In addition, the correction vector is removed. In this formulation, the number of free parameters is $P$. To further reduce complexity, $K$ eigenvectors $(K \leq P)$ can be obtained by PCA and used to replace $s_{p}$ in (22). In this case, there are only $K$ free parameters. It is reported in [61] that the ensemble modeling approach outperforms MLLR significantly on Aurora2 task. Two extensions of ensemble modeling are reported in [79]. The first extension is to use a tree-based clustering of the environments. During testing, the environment is first estimated, and only the supervectors of the selected environments are used for adaptation. The second extension is to use minimum classification error (MCE) criterion rather than ML $[99,100]$ to obtain discriminative supervectors.

\subsection{Environment Model-based Adaptation}

Another group of model adaptation methods uses the information of the environment model. A very brief review of such two popular methods, the Parallel Model Combination (PMC) [82] and Joint Compensation of Additive and Convolutive noise (JAC) [83] are given in the following sections.

\subsubsection{PMC}

Gales and Young [82] proposed the PMC approach, which synthesizes a noisy acoustic model using clean acoustic model and a noise model. In PMC, the noise is represented by a single or multi-state HMM depending on whether the noise is stationary. During the adaptation process, the noise model can be trained from the frames of silence segments in the testing utterances. Both the clean acoustic model and noise model are trained from cepstral features; however, the noisy acoustic model is synthesized in linear spectral domain, i.e. the filterbank domain before natural logarithm and DCT. Therefore, it is necessary to convert the mean and variance vectors of the clean and noise models back to the linear spectral domain first. After the noisy acoustic model is obtained in the linear spectral domain, it is then converted to the cepstral domain and used for speech recognition. The parameters of the clean acoustic model are compensated by adding the parameters of the noise model in the linear spectral domain. Specifically, for each clean and noise state pair, the mean vectors and covariance matrices of the two models are combined using the following formulae

$\hat{\mu}=g \mu+\bar{\mu}$

$\hat{\Sigma}=g^{2} \sum+\bar{\Sigma}$

where $(\hat{\mu}, \hat{\Sigma}),(\mu, \Sigma)$ are the noisy and clean speech model parameters, $\bar{\mu}, \bar{\Sigma}$ are the noise model parameters, all in linear spectral domain. The gain matching term $g$ is used, as the relative strengths of the speech and the noise in the testing environment may be different from these training environments, and it is estimated as

$g=\frac{E_{n s}-E_{n}}{E_{s}}$

where $E_{s}, E_{n s}$ and $E_{n}$ are the average energy of the clean speech, noisy speech and background noise respectively. It is reported in [82] that PMC significantly improves performance on isolated digit recognition task.

\subsubsection{JAC}

JAC [83] is another model adaptation method that uses an environment model. Similar to PMC, JAC also transforms the acoustic model's parameters back, but to log filterbank domain rather than linear spectral domain. Furthermore, JAC deals with both additive and convolutive noises, while original PMC only compensate for additive noise. In JAC, the phase-insensitive environment model is used. JAC first estimates the additive and convolutive noises from the current noisy test utterance using an EM algorithm. With these noise estimates, JAC adapts acoustic model's parameter in the log filterbank domain, and then converts the parameters back to cepstral domain. The adapted model is used to decode the test utterance, and the output alignment information is used to obtain a better estimation of the channel distortion, which is used as the initial value for the channel distortion for next test utterance. The additive noise estimate of current utterance is not similarly used in next test utterance as additive noise is assumed to be highly nonstationary. There are two extensions to JAC [85, 86]. In the first extension [85], vector Taylor series (VTS) is used to linearize the nonlinear distortion in the environment model. Another improvement is that the adaptation is now carried out in the cepstral domain directly and there is no need to convert the model parameters to log filterbank domain.

In addition, the extended JAC also adapts the Gaussian variances of the acoustic model, which are not adapted in original JAC. The second extension [86] is an improvement over the first extension. The major improvement is that the phaseinsensitive environment model is replaced by the phase-sensitive model for more accurate modeling of the relationship between noises and speech. It is reported in [86, 103] that the use of a phase-sensitive model significantly improves recognition performance on the Aurora- 2 task, where the data follows the phase-sensitive model well. 


\section{THE HYBRID MODEL}

The speech recognition is a pattern recognition task and Artificial Neural Network (ANN) is a good classifier. However, Artificial Neural Networks are not very good at handling dynamic data and speech signal is dynamic as it varies over time as it progresses. Thus, with standard neural networks it is difficult to handle this temporal variation in speech signals uttered by different speakers or at different speaking rates. Therefore, several researchers started combining elements of HMMs and neural networks [87 - 91, 101]. Various components of the HMM are implemented using neural networks to increase the performance of HMM system. In [90] a special recurrent neural network called a Viterbi Net was introduced as a way of implementing the Viterbi algorithm for an HMM. In [91] a similar model called recurrent AlphaNet, was introduced as a way of implementing the forward recursions of an HMM. Another very interesting neural network implementation of a discrete HMM is the so called Boltzmann chain introduced in [92]. The Boltzmann chain is a particular variant of Boltzmann machine [93], which is very similar to the globally normalized discrete HMM [93] that can be trained by a gradient-based algorithm which has many similarities to gradient-based forward-backward training for globally normalized HMMs.

So, rather than simply implementing the HMM alone, most of the current researchers also focus on HMM-ANN hybrids, to capitalize the strengths of each of the two frameworks: the temporal modeling capabilities of the HMM and the static classification or function approximation capabilities of Artificial Neural Networks. This hybrid model is also a trend towards efficient and robust system development in speech recognition research. However, it still remains a big challenge for the researchers to develop ASR system that gives 100\% accurate performance in mismatched environments or that matches the performance of human counterpart.

\section{CONCLUSION}

In this paper, major speech features used for speech recognition and different environment adaptation techniques for development for robust speech recognition system are reviewed. Integration of two different modeling techniques of HMM and Artificial Neural Networks to exploit the temporal modeling capabilities of HMM and the static classification capabilities of neural networks in order to construct robust speech recognition system is also reported. This paper may be useful as a tutorial and review on the state-of-the-art speech recognition systems, feature normalizations/adaptations, model adaptation etc. for students, scholars/researchers and other in the field of speech recognition research. It will be helpful for researches and development of robust ASR system in adverse environments.

\section{REFERENCES}

[1] B. H. Juang, and Lawrence R. Rabiner. "Automatic Speech Recognition - A brief History of the Technology Development.", Elsevier Encyclopedia of Language and Linguistics, 2004.

[2] Sadaoki Furui. "50 Years of Progress in Speech and Speaker Recognition.", ECTI Transactions on Computer and Information Technology, Vol. 1, No.2, November 2005.

[3] L. R. Rabiner and B. H. Juang. Fundamentals of Speech Recognition, Prentice-Hall, Englewood Cliff, New Jersey, 1993

[4] Tomi Kinnunen, Haizhou Li, "Overview of textindependent speaker recognition: From features to supervectors." Speech Communication, 52, pp. 12-40, 2010.

[5] D.S. Pallet, "Speech Results on Resource Management Task," in Proceedings of the February 1989 DARPA Speech and Natural Language Workshop, Morgan Kaufman Publishers, Inc., Philadelphia, PA, USA, pp. 1824, February 1989.

[6] D. Paul, "The Lincoln Robust Continuous Speech Recognizer," in Proceedings IEEE International Conference on Acoustics, Speech, and Signal Processing, pp. 556-559, Glasgow, Scotland, May 1989.

[7] J.G. Wilpon, R.P. Mikkilineni, D.B. Roe, and S. Gokcen, "Speech Recognition: From the Laboratory to the Real World," AT\&T Technical Journal, vol. 69, no. 5, pp. 14-24, October 1990.

[8] J.G. Wilpon, D.M. DeMarco, R.P. Mikkilineni, "Isolated Word Recognition Over the DDD Telephone Network Results Of Two Extensive Field Trials," in Proceedings IEEE International Conference on Acoustics, Speech, and Signal Processing, pp. 55-57, New York, NY, USA April 1988.

[9] B. Wheatley and J. Picone, "Voice Across America: Toward Robust Speaker Independent Speech Recognition For Telecommunications Applications", Digital Signal Processing: A Review Journal, vol. 1, no. 2, p. 45-64, April 1991.

[10] Picone, J., "The Demographics of Speaker Independent Digit Recognition", in Proceedings IEEE International Conference on Acoustics, Speech, and Signal Processing, pp. 105-108, Albuquerque, New Mexico, USA, April 1990.

[11] Jain, A., Duin, R., Mao, J., "Statistical pattern recognition: a review." IEEE Transaction on Pattern Anal. Machine Intelligence, 22 (1), 4-37, 2000

[12] Furui, S., "Cepstral analysis technique for automatic speaker verification." IEEE Trans. Acoustics, Speech Signal Process, 29 (2), 254-272, 1981.

[13] Huang, X., Acero, A.,H. W., Hon, Spoken Language Processing: a Guide to Theory, Algorithm, and System Development, Prentice-Hall, New Jersey.

[14] S. Dupont, et. al. "Hybrid HMM/NN Systems for Training Independent Tasks: Experiments on Phonebook and Related Improvements.", Proceedings of International Conference on Acoustics, Speech and Signals, pp. 1767 1770, Munich, Germany, April 1997.

[15] J. Hennebert, C. Ris, H. Bourlard, S. Renals, and N. Morgan. "Estimation of Global Posteriors and ForwardBackward Training of Hybrid HMM/NN Systems.", Proceedings of EUROSPEECH'97, 1997.

[16] Iain Matthews et al., "Extraction of Visual Features for Lipreading.", IEEE Transaction on Pattern Analysis and Machine Intelligence, vol. 24, No. 2, February 2002.

[17] T.F. Cootes, G.J. Edwards, and C.J. Taylor, "Active Appearance Models," Proc. European Conf. Computer Vision, pp. 484-498, June 1998.

[18] T.F. Cootes, C.J. Taylor, D.H. Cooper, and J. Graham, "Active Shape Models-Their Training and Application,' 
Computer Vision and Image Understanding, vol. 61, no. 1, pp. 38-59, Jan. 1995.

[19] R. P. Lipmann. "Speech Recognition by Machines and Humans.", Speech Communication, vol. 22, pp. 1 - 15, 1997.

[20] J. S. Lim and A. V. Oppenheim, "Enhancement and bandwidth compression of noisy speech," Proc. IEEE, vol. 67, pp. 1586-1604, Dec. 1979.

[21] R. J. Mcaulay and M. L. Malpass, "Speech enhancement using a soft-decision noise suppression filter," IEEE Trans. Acoustics, Speech and Signal Processing, vol. 28, pp. 137145, Apr. 1980.

[22] S. F. Boll, "Suppression of acoustic noise in speech using spectral subtraction," IEEE Trans. Acoustics, Speech and Signal Processing, vol. 27, no. 2, pp. 113-120, 1979.

[23] M. Berouti, R. Schwartz, and J. Makhoul, "Enhancement of speech corrupted by acoustic noise," in Proc. ICASSP '79, USA, pp. 208-211, Apr. 1979.

[24] N. Virag, "Single channel speech enhancement based on masking properties of the human auditory system," IEEE Trans. Speech and Audio Processing, vol. 7, pp. 126-137, Mar. 1999.

[25] L. Deng and X. Huang, "Challenges in adopting speech recognition," Communications of the ACM, vol. 47, no. 1, pp. 69-75, 2004.

[26] P. Lockwood and J. Boudy, "Experiments with a nonlinear spectral subtractor (NSS), Hidden Markov Models and projection, for robust recognition in cars," Speech Communication, vol. 11, no. 2-3, pp. 215-228, 1992.

[27] Y. Ephraim and D. Malah, "Speech enhancement using a minimum mean square error short time spectral amplitude estimator," IEEE Trans. Acoustics, Speech and Signal Processing, vol. 32, pp. 1109-1121, Dec. 1984.

[28] Y. Ephraim and D. Malah, "Speech enhancement using a minimum mean square error log-spectral amplitude estimator," IEEE Trans. Acoustics, Speech and Signal Processing, vol. 33, pp. 443-445, Apr. 1985.

[29] I. Y. Soon and S. N. Koh, "Low distortion speech enhancement," IEE Proc. On Visual Image Signal Processing, vol. 147, pp. 247-253, Jun. 2000.

[30] M. K. Hasan, S. Salahuddin, and M. R. Khan, "A modified a priori SNR for speech enhancement using spectral subtraction rules," IEEE Signal Processing let ters, vol. 11, pp. 450-453, Apr. 2004.

[31] I. Cohen, "Speech enhancement using a non causal A Priori SNR estimator," IEEE Signal Processing letters, vol. 11, pp. 725-728, Sep. 2004.

[32] Y. Hu and P. C. Loizou, "Speech enhancement based on wavelet thresholding the multitaper spectrum," IEEE Trans. Speech and Audio Processing, vol. 12, pp. 59-67, Jan. 2004.

[33] H. K. Kim and R. Rose, "Cepstrum-domain acoustic feature compensation based on decomposition of speech and noise for ASR in noisy environments," IEEE Trans. Speech and Audio Processing, vol. 11, pp. 435-446, Sept. 2003.
[34] R. Gemello, F. Mana, and R. De Mori, "Automatic speech recognition with a modi_ed ephraim-malah rule," IEEE Signal Processing letters, vol. 13, pp. 56-59, Jan. 2006.

[35] M. Dendrinos, S. Bakamidis, and G. Carayannis, "Speech enhancement from noise: A regenerative approach," Speech Communication, vol. 10, pp. 45-57, Feb. 1991.

[36] S. H. Jensen, P. C. Hansen, S. D. Hansen, and J. A. Soensen, "Reduction of broad-band noise in speech by truncated QSVD," IEEE Trans. Speech and Audio Processing, vol. 3, pp. 439-444, Nov. 1995.

[37] Y. Ephraim and H. L. Van Trees, "A signal subspace approach for speech enhancement," IEEE Trans. Speech and Audio Processing, vol. 3, pp. 251-266, Jul. 1995.

[38] J. Huang and Y. Zhao, "An energy-constraind signal subspace method for speech enhancement and recognition in colored noise," in Proc. ICASSP '98, vol. 1, pp. 377380, May 1998.

[39] A. Rezayee and S. Gazor, "An adaptive KLT approach for speech enhancement,"IEEE Trans. Speech and Audio Processing, vol. 9, pp. 87-95, Feb. 2001.

[40] K. Hermus and P.Wambacq, "Assessment of signal subspace based speech enhancement for noise robust speech recognition," Acoustics, Speech, and Signal Processing, 2004. Proceedings. (ICASSP '04), IEEE International Conference on, vol. 1, pp. I-45-8, May 2004.

[41] U. Mittal and N. Phamdo, "Signal/noise KLT based approach for enhancing speech degraded by colored noise," IEEE Trans. Speech and Audio Processing, vol. 8, pp. 159167, Mar. 2000.

[42] A. Acero and R. M. Stern, "Environmental robustness in Automatic Speech Recognition." In Proc. IEEE Acoustics, Speech and Signal Processing, pp. 849-582. April 1990.

[43] A. P. Dempster, N. M. Laird, and D. B. Rubin, "Maximum likelihood from incomplete data via the em algorithm," Journal of Royal Statistical Society, Series B (Methodological), vol. 39, no. 1, pp. 1-38, 1977.

[44] L. Deng, J. Droppo, and A. Acero, "Enhancement of log mel power spectra of speech using a phase-sensitive model of the acoustic environment and sequential estimation of the corrupting noise," IEEE Trans. Speech and Audio Processing, vol. 12, pp. 133-143, Mar. 2004.

[45] L. Deng, J. Droppo, and A. Acero, "Estimating cepstrum of speech under the presence of noise using a joint prior of static and dynamic features," IEEE Trans. Speech and Audio Processing, vol. 12, pp. 218-223, May 2004.

[46] L. Deng, J. Droppo, and A. Acero, "Dynamic compensation of HMM variances using the feature enhancement uncertainty computed from a parametri model of speech distortion," IEEE Trans. Speech and Audio Processing, vol. 13, pp. 412-421, May 2005.

[47] L. Deng, J. Droppo, and A. Acero, "Recursive estimation of nonstationary noise using iterative stochastic approximation for robust speech recognition," IEEE Trans.Speech and Audio Processing, vol. 11, pp. 568-580, Nov. 2003. 
[48] L. Deng, J. Droppo, and A. Acero, “A Bayesian approach to speech feature enhancement using the dynamic cepstral prior," In Proc. ICASSP '02, (Orlando,USA), May 2002.

[49] L. Buera, E. Lleida, A. Miguel, A. Ortega, and O. Saz, "Cepstral vector normalization based on stereo data for robust speech recognition," IEEE Trans. Audio, Speech, and Language Processing, vol. 15, pp. 1098-1113, March 2007.

[50] P. J. Moreno, B. Raj, and R. M. Stern, "Data-driven environmental compensation for speech recognition: A unified approach," Speech Communication, vol. 24, pp. 267-285, Jul. 1998.

[51] L. Deng, A. Acero, M. Plumpe, and X. D. Huang, "Largevocabulary speech recognition under adverse acoustic environment," in Proc. ICSLP '00, pp. 806-809, China.

[52] L. Deng, A. Acero, L. Jiang, J. Droppo, and X. D. Huang, "High-performance robust speech recognition using stereo training data," in Proc. ICASSP '01, USA), pp. 301-304, May 2001.

[53] J. Droppo, L. Deng, and A. Acero, IEvaluation of the SPLICE algorithm on the Aurora2 database," in Proc. Eurospeech '01, (Aalborg, Denmark), pp. 217-220,Sept. 2001

[54] J. Droppo, L. Deng, and A. Acero, "Uncertainty decoding with SPLICE for noise robust speech recognition," in Proc. ICASSP '02, (Orlando, USA), May 2002.

[55] F. H. Liu, R. M. Stern, X. Huang, and A. Acero, "Efficient cepstral normalization for robust speech recognition," in Proc. ARPA Human Language Technology Workshop '93, (Princeton, NJ), pp. 69-74, Mar. 1993.

[56] L. Wang, N. Kitaoka, and S. Nakagawa, "Robust distant speech recognition by combining multiple microphonearray processing with position-dependent CMN," EURASIP Journal on Applied Signal Processing, vol. 2006, pp. 1-11, 2006

[57] O. Viikki and K. Laurila, "Cepstral domain segmental feature vector normalization for noise robust speech recognition," Speech Communication, vol. 25, pp. 133-147, 1998

[58] F. Hilger and H. Ney, "Quantile based histogram equalization for noise robust large vocabulary speech recognition," IEEE Trans. Audio, Speech, and Language Processing, vol. 14, no. 3, pp. 845-854, 2006.

[59] A. de la Torre, A. M. Peinado, J. C. Segura, J. L. PerezCordoba, M. C. Benitez, and A. J. Rubio, "Histogram equalization of speech representation for robust speech recognition," IEEE Trans. Speech and Audio Processing, vol. 13 , no. 3 , pp. 355-366, 2005

[60] Y. Suh, M. Ji, and H. Kim, "Probabilistic class histogram equalization for robust speech recognition," IEEE Signal Processing letters, vol. 14, no. 4, pp. 287-290, 2007.

[61] Xiong Xiao, Eng Siong Chng and Haizhou Li, "Normalizing the speech modulation spectra for robust speech recognition," IEEE Transactions on Audio, Speech, and Language processing, vol. 16, no. 8, pp. 1662-1674, November 2008.
[62] T. Houtgast and H. J. M. Steeneken, "The modulation transfer function in room acoustics as a predictor of speech intelligibility," Acustica, vol. 28, pp. 66-73, 1973.

[63] T. Houtgast and H. J. M. Steeneken, "A review of the MTF concept in room acoustics and its use for estimating speech intelligibility in auditoria," Journal of the Acoustical Society of America, vol. 77, no. 3, pp. 1069-1077, 1985.

[64] R. Drullman, J. M. Festen, and R. Plomp, "Effect of temporal envelope smearing on speech reception," Journal of the Acoustical Society of America, vol. 95, no. 2, pp. 1053-1064, 1994.

[65] R. Drullman, J. M. Festen, and R. Plomp, "Effect of reducing slow temporal modulations on speech reception," Journal of the Acoustical Society of America, vol. 95, no. 5, pp. 2670-2680, 1994.

[66] N. Kanedera, T. Arai, H. Hermansky, and M. Pavel, "On the relative importance of various components of the modulation spectrum for automatic speech recognition," Speech Communication, vol. 28, no. 1, pp. 43-55, 1999.

[67] T. Arai, M. Pavel, H. Hermansky, and C. Avendano, "Syllable intelligibility for temporally filtered LPC cepstral trajectories," Journal of the Acoustical Society of America, vol. 105, no. 5, pp. 2783-2791, 1999.

[68] C. P. Chen and J. A. Bilmes, "MVA processing of speech features," IEEE Trans. Audio, Speech, and Language Processing, vol. 15, no. 1, pp. 257-270, 2007.

[69] C. Avendano, S. van Vuuren, and H. Hermansky, "Data based filter design for RASTA-like channel normalization in ASR," in Proc. ICSLP '96, (Philadelphia, USA), Oct. 1996.

[70] S. van Vuuren and H. Hermansky, "Data-driven design of RASTA-like filters," in Proc. Eurospeech '97, Sept. 1997.

[71] H. Hermansky, "The modulation spectrum in the automatic recognition of speech," in Proc. ASRU '97, pp. 140-147, Dec 1997.

[72] M. Shire and B. Chen, IOn data-derived temporal processing in speech feature extraction," in Proc. ICSLP '00, (Beijing, China), Oct. 2000.

[73] M. L. Shire, "Data-driven modulation_lter design under adverse acoustic conditions and using phonetic and syllabic units," in Proc. Eurospeech '99, (Budapest, Hungary), Sept 1999.

[74] J.-W. Hung and L.-S. Lee, "Optimization of temporal filters for constructing robust features in speech recognition," IEEE Trans. Audio, Speech, and Language Processing, vol. 14, no. 3, pp. 808-832, 2006.

[75] A. Sankar and C.-H. Lee, "A maximum-likelihood approach to stochastic matching for robust speech recognition," IEEE Trans. Speech and Audio Processing, vol. 4, pp. 190-202, May 1996.

[76] A. Surendran, C.-H. Lee, and M. Rahim, "Nonlinear compensation for stochastic matching," Speech and Audio Processing, IEEE Transactions, 7, pp. 643-655, Nov 1999.

[77] C.-S. Huang, W. Hsiao-Chuan, and C.-H. Lee, "An snrincremental stochastic matching algorithm for noisy speech recognition," Speech and Audio Processing, IEEE Transactions vol. 9, pp. 866-873, Nov 2001. 
[78] J. L. Gauvain and C. H. Lee, "Maximum a posterirori estimation for multivariate Gaussian mixture observations of Markov chains," IEEE Trans. Speech and Audio Processing, vol. 2, pp. 291-298, Apr. 1994.

[79] Q. Huo, C. Chan, and C. H. Lee, "Bayesian adaptive learning of the parameters of hidden Markov model for speech recgonition," IEEE Trans. Speech and Audio Processing, vol. 3, pp. 334-345, Sep. 1995.

[80] Y. Tsao and C.-H. Lee, "Two extensions to ensemble speaker and speaking environment modeling for robust automatic speech recognition," in Proc. ASRU '07, pp. 7780, Dec. 2007.

[81] Y. Tsao and C.-H. Lee, “An ensemble modeling approach to joint characterization of speaker and speaking environments," in Proc. Eurospeech '07, pp. 1050-1053, Sept. 2007.

[82] R. Kuhn, J.-C. Junqua, P. Nguyen, and N. Niedzielski, "Rapid speaker adaptation in eigenvoice space," IEEE Trans. Speech and Audio Processing, vol. 8, pp. 695-707, Nov 2000.

[83] M. J. F. Gales and S. J. Young, "Cepstral parameter compensation for HMM recognition," Speech Communication, vol. 12, pp. 231-239, Jul. 1993.

[84] Y. Gong, "A method of joint compensation of additive and convolutive distortions for speaker-independent speech recognition," IEEE Trans. Speech and Audio Processing, vol. 13, no. 5, pp. 975-983, 2005.

[85] T. Takiguchi, S. Nakamura, and K. Shikano, "Hmmseparation-based speech recognition for a distant moving speaker," Speech and Audio Processing, IEEE Transactions, vol. 9, pp. 127-140, Feb 2001.

[86] J. Li, L. Deng, D. Yu, Y. Gong, and A. Acero, "Highperformance HMM adaptation with joint compensation of additive and convolutive distortions via vector taylor series," in Proc. ASRU '07, (Kyoto, Japan), pp. 65-70, Dec. 2007.

[87] J. Li, L. Deng, D. Yu, Y. Gong, and A. Acero, "HMM adaptation using a phase sensitive acoustic distortion model for environment-robust speech recognition," in Proc. ICASSP '08, pp. 4069-4072, Apr. 2008.

[88] D. P. Morgan and C. L. Scofield. Neural Networks for Speech Processing. Kluwer Academic Publishers, Norwell, Massachussets, 1991.

[89] S. Riis, "Hidden Neural Network: Application to speech recognition", In proceedings of Int. Conf. on Acoustics, Speech and Signal Processing, Seatle, 1998.

[90] Lipmann, R. P. and Gold, B., "Neural net classifiers useful for speech recognition, In Proceedings of IEEE first International Conference on Neural Networks, vol. IV, pp. 417-425, June 1987.
[91] Briddle, J. S., "Alphanets: a recurrent neural network architecture with a hidden markov model interpretation, Speech Communication, 9:83-92, Feb. 1990.

[92] Saul, L. K. and Jordan, M. I., "Boltzmann Chains and Hidden markov models, In Advances in Neural Information Processing Systems, vol. 7, pp. 435-442, 1995.

[93] Ackley, D. H., Hinton, G. E. and Sejnowjki, T. J., "A learning algorithm for Boltzmann machine", Cognitive Science, 9:147-169, 1985.

[94] Mackay, D. J. C., "Equivalence of linear Boltzmann chains and hidden markov models. Neural computation, 1(8):178$181,1996$.

[95] S. Furui. "Speaker independent isolated word recognition using dynamic features of speech spectrum.", IEEE Trans. Acoustics, Speech, Signal Processing, ASSP-34, pp. 52-59, 1986.

[96] H. Hermansky, and N. Morgan. "RASTA-Processing of Speech.”, IEEE Transactions on Speech and Audio Processing, vol. 2, No.4, pp. 578 - 589, 1994.

[97] K. Shikano, "Evaluation of LPC Spectral Matching Measures for Phonetic Unit Recognition," TM No. CMU CS-86- 108, Computer Science Department, CarnegieMellon University, Pittsburgh, PA, US, 15213, February 3, 1986.

[98] C. J. Leggetter and P. C. Woodland. "Maximum Likelihood Linear Regression for Speaker Adaptation of Continuous Density Hidden Markov Models", Computer Speech and Language, vol. 9, pp. 171 - 185, 1995.

[99] B. H. Juang, C. H. Lee, and Wu Chou. "Minimum Classification Error Rate Methods for Speech Recognition.", IEEE Transactions on Speech \& Audio Processing, T-SA, vol. 5, No. 3, pp. 257 - 265, May 1997.

[100] B. H. Juang and S. Katagiri. "Discriminative Learning for Minimum Error Classification.", IEEE Trans. Signal Processing, vol. 40, pp. 3043-3054, 1992.

[101] R. P. Lipmann. "Review of Neural Networks for Speech Recognition.”, Neural Computation, 1, 1-38, 1989.

[102] M.A. Anusuya · S.K. Katti, "Front end analysis of speech recognition: a review", Int J Speech Technology, 14: 99$145,2011$.

[103]http://www3.ntu.edu.sg/home/xiaoxiong/TsceG0402426L. pdf 